\title{
Association of supraclavicular node metastasis with survival in node positive esophageal squamous cell carcinoma patients treated using definitive chemoradiation
}

\author{
Jia-Hua Yen ${ }^{1}$, Chung-Wen Jen ${ }^{1}$, Tzu-Ting Huang ${ }^{2}$, Yu-Chen Tsai ${ }^{1}$, Skye Hung-Chun Cheng ${ }^{1,3}$ \\ ${ }^{1}$ Department of Radiation Oncology, Koo Foundation Sun Yat-Sen Cancer Center, Taipei, Taiwan; ${ }^{2}$ Department of Research, Koo Foundation Sun \\ Yat-Sen Cancer Center, Taipei, Taiwan; ${ }^{3}$ Departement of Radiation Oncology, Taitung Christian Hospital, Taitung, Taiwan \\ Contributions: (I) Conception and design: JH Yen, CW Jen, SHC Cheng; (II) Administrative support: YC Tsai, SHC Cheng; (III) Provision of study \\ materials or patients: YC Tsai, SHC Cheng; (IV) Collection and assembly of data: JH Yen, CW Jen; (V) Data analysis and interpretation: CW Jen, \\ TT Huang; (VI) Manuscript writing: All authors; (VII) Final approval of manuscript: All authors. \\ Correspondence to: Skye Hung-Chun Cheng. Department of Radiation Oncology, Koo Foundation Sun Yat-Sen Cancer Center, 125 Lih-Der Road, \\ Pei-Tou District, Taipei, Taiwan. Email: Skye@kfsyscc.org.
}

Background: The study aimed to assess the prognostic significance of supraclavicular lymph node (SCLN) metastasis in patients with node-positive esophageal squamous cell carcinoma (ESCC) treated using definitive chemoradiation.

Methods: A total of 143 patients with newly diagnosed ESCC treated using definitive chemoradiation between 2006 and 2017 were identified retrospectively in our observational cohort study. Data for clinical characteristics, including the gross tumor volume (GTV) and radiation dose were collected. Among these patients, 71 had SCLN metastasis. Data of additional 80 ESCC patients with de novo distant metastasis were collected. Survival and failure estimates were calculated according to the methods of Kaplan-Meier. Prognostic factors were evaluated using multivariate analyses.

Results: The median follow-up time was 14.3 months (range, 1.9-139.3 months) and the median overall survival (OS) of the entire cohort was 16.1 months [95\% confidence interval (CI), 12.9-20.7 months]. The 2 -year survival rates were similar in patients with and without SCLN metastasis $(32.8 \%, \mathrm{P}=0.88)$. Time to failure was also similar between the two groups $(\mathrm{P}=0.54)$. There was a significant 2 -year OS difference between patients with de novo distant metastasis and SCLN metastasis $(\mathrm{P} \leq 0.00071)$. The multivariate analysis demonstrated that SCLN metastasis was not associated with poorer survival. Higher radiation dose and lower pretreatment neutrophil-lymphocyte ratio were independently associated with better OS.

Conclusions: For patients with ESCC treated with definitive chemoradiation, SCLN metastasis was not independently associated with poorer survival. Curative treatment should be considered for these patients. Higher radiation dose and lower pretreatment neutrophil-lymphocyte ratio improved the treatment outcomes. Keywords: Supraclavicular lymph node metastasis (SCLN metastasis); chemoradiation; esophageal squamous cell
carcinoma (ESCC); prognosis; staging system

Received: 15 June 2020; Accepted: 11 September 2020; Published: 30 December 2020.

doi: $10.21037 /$ tro-20-45

View this article at: http://dx.doi.org/10.21037/tro-20-45

\section{Introduction}

Esophageal squamous cell carcinoma (ESCC) is the most common type of esophageal cancer in Asia. Despite advances in the treatment modalities, the aggressive nature of esophageal cancer still results in poor prognosis, with the 5-year survival rate being approximately $30-40 \%$ (1). 
The presence of regional lymph node involvement is one of the most important prognostic factors. The incidence and distribution pattern of lymphatic drainage are influenced by various factors, including the location of the primary tumor, $\mathrm{T}$ category, and histology (2-4). Currently, the 8th edition of the Union for International Cancer Control-American Joint Committee on Cancer (UICC-AJCC) TNM staging system for esophageal cancer defines lymph nodes located in the continuity with the esophagus as regional lymph nodes. It states that station 1 contains lower cervical paratracheal nodes between the supraclavicular paratracheal space and the apex of the lung. Therefore, the supraclavicular lymph node (SCLN) is considered a non-regional lymph node in the current UICC-AJCC staging system (5). In contrast, according to the 7 th edition of the Japanese Classification of Esophageal Cancer by the Japan Esophageal Society (JES), the primary tumor location influences the definition of regional lymph nodes (6). SCLN metastasis is considered a stage IV disease for tumors originating from the abdominal esophagus alone.

A surgical series from Japan demonstrated that patients with SCLN metastasis can be long-term survivors after curative intent resection. Compared with patients with the same number of lymph nodes involved, patients with SCLN metastasis had similar outcomes (7). Moreover, several studies have also demonstrated that patients with SCLN metastasis have better survival rates than patients with visceral metastasis $(8,9)$. For patients who cannot tolerate surgery or refuse surgery, definitive chemoradiation is the standard approach. This study aimed to investigate the prognostic significance of SCLN metastasis in patients with unresectable ESCC receiving definitive chemoradiation for overall survival (OS). We present the following article in accordance with the STROBE reporting checklist (available at http://dx.doi.org/10.21037/tro-20-45).

\section{Methods}

\section{Patients}

The study was conducted in accordance with the Declaration of Helsinki (as revised in 2013). The study was approved by the institutional ethics board (No.: 20190220A). All the patients have given a consent for medical record collection before receiving treatment. There is no specified informed consent for this study. All the information obtained only includes routine clinical practice. No additional management was done for patients who were enrolled in this study. In this cohort study, we retrospectively collected data of patients with ESCC treated using chemoradiation at our cancer center from 2006 to 2017. We excluded patients with visceral or bone metastatic disease, node-negative disease, previous or synchronous cancer, planned esophagectomy, radiation dose less than $50 \mathrm{~Gy}$, which was not considered as definitive treatment, and those with incomplete clinical or radiotherapy information. Figure 1 shows the detailed information of study flow; a total of 143 patients were identified for this study.

Patients were considered non-surgical candidates following the discussion with a multidisciplinary team. The diseases were considered unresectable because of radiographic or endoscopic findings of cervical esophagus involvement, it was deemed medically inoperable, or the patient declined surgery. We defined clinical nodal positivity by either positron emission tomography-computed tomography (PET-CT) scan with ${ }^{18} \mathrm{~F}$-fluorodeoxyglucose (FDG) uptake of involved nodes or with nodes larger than $10 \mathrm{~mm}$ in maximum transverse diameter on diagnostic computed tomography (CT) scan. Among the SCLN metastasis group, tissue diagnosis was performed in 20 patients $(28.1 \%)$. Biopsy was not performed in the others for the following reasons: proximity of critical vessels, inconclusive biopsy, imaging was deemed sufficient, and other potential morbidities. The consensus clinical staging before treatment of each our patient was reached after multidisciplinary team discussion. We also collected clinical information and treatment characteristics including age, sex, $\mathrm{T}$ and $\mathrm{N}$ staging, performance status, primary tumor location, radiation dose, gross tumor volume (GTV), and neutrophil-lymphocyte ratio (NLR). NLR was defined as neutrophil counts divided by lymphocyte counts before treatment. The anatomical boundary of SCLN metastasis was defined by the common carotid artery and internal jugular vein medially, sternocleidomastoid muscle anteriorly and laterally, and scalene muscle posteriorly (10). During the same study period, an additional 80 ESCC patients with de novo distant metastasis were identified. The metastatic site included visceral organs, bone, and non-regional lymph nodes such as the neck, retroperitoneal, or axilla.

\section{Treatment}

Radiation therapy was delivered either by 3-dimensional conformal radiation therapy (3D CRT) or intensitymodulated radiation therapy (IMRT) with weekly image guidance. GTV was defined as the gross tumor or lymph 


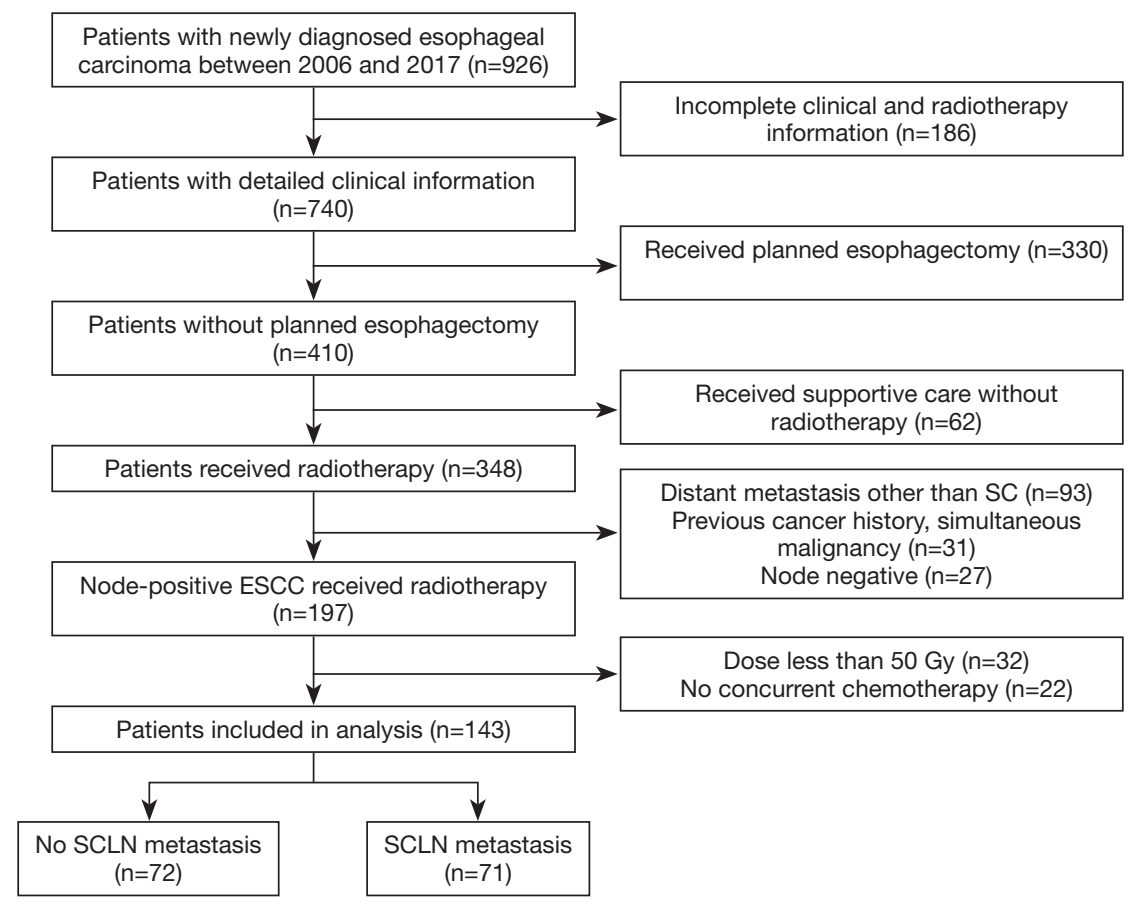

Figure 1 Consolidated standards of reporting trials (CONSORT) flow diagram showing patient selection. SCLN, supraclavicular lymph node; SC, supraclavicular.

nodes noted by all diagnostic modalities. The mediastinal and lymph node regions at risk were included in the clinical target volume (CTV). A 1-2 cm margin was expanded from the GTV and CTV to generate the planning target volume (PTV). A radiation dose of 50-70 Gy was prescribed if normal organ constraints were met. Most patients received concurrent chemotherapy with cisplatin $(75 \mathrm{mg} / \mathrm{m} 2)$ on day 1 and 5 -fluorouracil (1,000 $\mathrm{mg} / \mathrm{m} 2$; continuous infusion) on days 1-4 every 4 weeks for two cycles. CT scan and esophagogastroduodenoscopy were performed within 3 months after the completion of chemoradiation. Any suspicious lesions would be biopsied to screen for viable tumors. We defined patients without therapeutic response as progressive local disease, persistent local disease, or new distant metastasis with the help of an experienced diagnostic radiologist. All patients were reviewed clinically every 3 months for 2 years, every 6 months in the third to fifth year, and once a year thereafter. Esophagogastroduodenoscopy and CT scan were performed every 6 months for surveillance. Local failure was defined as recurrence within the PTV. Recurrence or progression found in visceral organs, neck lymph nodes other than SCLN, and abdominal para-aortic lymph nodes were considered as distant failures. Patients with failure both locally and distantly were considered to have synchronous failures.

\section{Statistical analysis}

OS was defined as the time from treatment to death from any cause. The last follow-up time was on December 31, 2017. We also incorporated the survival database from the Taiwan National Cancer Registry for patients who did not have regular follow-up at our hospital. Patient demographics were compared using independent KruskalWallis test for continuous parameters and the Chi-square test or Fisher's exact test for categorical parameters. Crude estimates of OS and progression were derived using KaplanMeier methods. The log-rank test was used to compare the survival curves of different patient groups. Significant variables in univariate analysis and in previous reports were included in the multivariate analysis. The association of each variable with OS was derived from the multivariate Cox proportional hazards model. The statistical significance of each of the variables included in the model was assessed using likelihood ratio tests. No missing data were observed in collected data. The Chi-square test was used to evaluate differences in failure patterns between the SCLN metastasis group and the no SCLN metastasis group. We converted 
Table 1 Baseline characteristics of patients with or without supraclavicular lymph node (SCLN) metastasis

\begin{tabular}{|c|c|c|c|}
\hline Characteristic & $\begin{array}{l}\text { No SCLN mets } \\
\qquad(\mathrm{n}=72)\end{array}$ & $\begin{array}{l}\text { SCLN mets } \\
\quad(n=71)\end{array}$ & $P$ value \\
\hline Sex & & & 0.7721 \\
\hline Female & $6(8.3 \%)$ & $5(7.0 \%)$ & \\
\hline Male & $66(91.7 \%)$ & $66(93.0 \%)$ & \\
\hline Age & & & 0.637 \\
\hline Mean (SD) & $58.8(10.5)$ & $57.6(9.37)$ & \\
\hline T category & & & 0.872 \\
\hline T1-2 & $5(6.9 \%)$ & $4(5.6 \%)$ & \\
\hline T3 & $53(73.6 \%)$ & $55(77.5 \%)$ & \\
\hline $\mathrm{T} 4$ & $14(19.4 \%)$ & $12(16.9 \%)$ & \\
\hline $\mathrm{N}$ category & & & $<0.001^{*}$ \\
\hline $\mathrm{N} 1$ & $50(69.4 \%)$ & $21(29.6 \%)$ & \\
\hline N2-3 & $22(30.6 \%)$ & $50(70.4 \%)$ & \\
\hline Performance status & & & 0.5753 \\
\hline ECOG 0-1 & $65(90.3 \%)$ & $62(87.3 \%)$ & \\
\hline ECOG 2-3 & $7(9.7 \%)$ & $9(12.7 \%)$ & \\
\hline Primary tumor location & & & $0.0451^{*}$ \\
\hline Cervical & $13(18.1 \%)$ & $9(12.7 \%)$ & \\
\hline Upper thoracic & $10(13.9 \%)$ & $15(21.1 \%)$ & \\
\hline Middle thoracic & $22(30.6 \%)$ & $33(46.5 \%)$ & \\
\hline Lower thoracic & $27(37.5 \%)$ & $14(19.7 \%)$ & \\
\hline \multicolumn{4}{|l|}{ Radiation dose } \\
\hline Mean (SD, Gy) & $58.3(5.31)$ & $58.1(4.75)$ & 0.734 \\
\hline \multicolumn{4}{|l|}{ NLR } \\
\hline Mean (SD) & $5.04(3.93)$ & $4.81(4.73)$ & 0.47 \\
\hline \multicolumn{4}{|l|}{ GTV } \\
\hline Mean (SD, cc) & $109(71.7)$ & $126(102)$ & 0.408 \\
\hline
\end{tabular}

Chi-square test or Fisher's exact test was used for categorical variables and the Kruskal-Wallis test was used for continuous variables. The $\mathrm{T}$ and $\mathrm{N}$ categories were analyzed based on the AJCC 8th edition staging system. AJCC, American Joint Committee on Cancer; ECOG, Eastern Cooperative Oncology Group; NLR, Neutrophil-to-lymphocyte ratio; GTV, gross tumor volume.

the radiation dose from a continuous variable into a categorical variable to establish the optimal radiation dose for these patients. Kaplan-Meier survival curves were generated in R (version

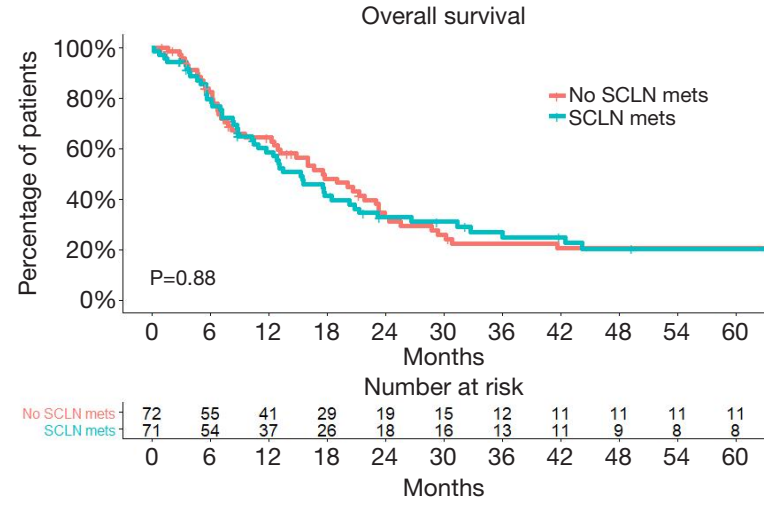

Figure 2 Survival curve of node-positive esophageal cancer patients with or without supraclavicular lymph node metastasis. SCLN metastasis, supraclavicular lymph node metastasis.

3.4.1; www.r-project.org). All tests were two-sided, and a $\mathrm{P}$ value $<0.05$ was considered statistically significant.

\section{Results}

\section{Baseline characteristics}

A total of 143 patients received chemoradiation for nonmetastatic inoperable ESCC between 2006 and 2017 in our institution were identified. The median follow-up time was 14.3 months for patients alive (range, 1.9-139.3 months) and the median OS of the entire cohort was 16.1 months [95\% confidence interval (CI), 12.9-20.7 months]. The median age was 57.5 years old (range, 37.6-84.7 years). Among these patients, $92.36 \%$ were male. The median radiation dose was 59.4 Gy (range, 50-70 Gy). Seventyone patients had SCLN metastasis. Patient characteristics in the SCLN metastasis group and the no SCLN metastasis group are listed in Table 1. The nodal staging was more advanced, and the primary tumor was more common in the upper/middle thoracic esophagus in patients with SCLN metastasis. Other baseline characteristics were similar.

Although patients with SCLN metastasis had more advanced nodal staging, the status of SCLN metastasis did not significantly affect the OS in these node-positive patients.

The Kaplan-Meier OS curves for both groups are shown in Figure 2. The 2-year survival rate was $32.8 \%$ (95\% CI, 23.1-46.7\%) for patients with SCLN metastasis and 32.8\% (95\% CI, 23.0-47.0\%) for those without SCLN metastasis $(\mathrm{P}=0.88)$. 


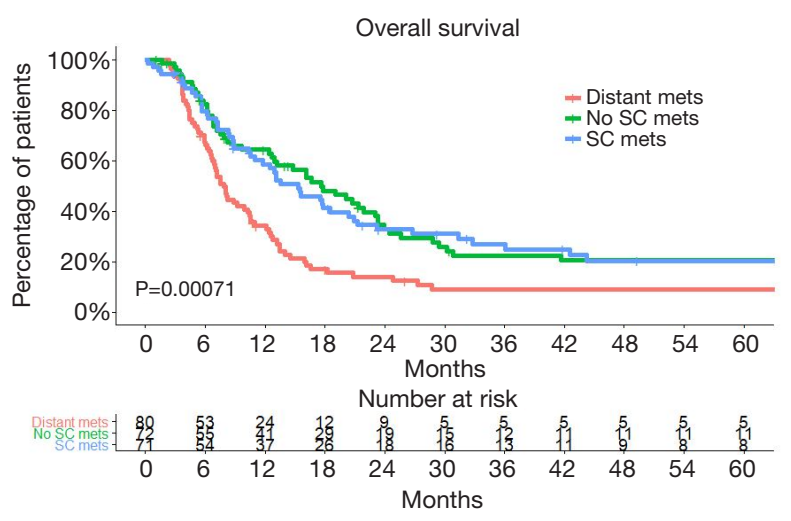

Figure 3 Survival curve of patients with de novo distant metastasis esophageal cancer. SC metastasis, supraclavicular metastasis.

A significant 2-year OS difference between patients with de novo distant metastasis and those with SCLN metastasis was also noted in our hospital $(\mathrm{P}<0.00071)$. The 2 -year survival rate was $14.0 \%$ (95\% CI, 7.9-24.9\%) for patients with de novo distant metastasis (Figure 3).

Further, 26 patients did not show therapeutic response after treatment in our study. The median OS for patient with therapeutic responses after treatment in our cohort was 20.7 months (95\% CI, 17.5-24.4 months) and 6.8 months (95\% CI, 6.1-8.8 months) for those without therapeutic responses.

\section{Multivariate analysis of $O S$}

We performed a multivariate analysis using the Cox proportional hazard model. T1 and T2 were analyzed as one group because of the small numbers in T1. Similarly, the $\mathrm{N}$ category was divided into two groups, N1 and $\mathrm{N} 2 / 3$. The multivariate survival analysis demonstrated that SCLN metastasis was not associated with poorer survival $(\mathrm{P}=0.837)$. Prognostic factors for OS in the multivariate survival analysis were pretreatment NLR (as a continuous variable, $\mathrm{P}=0.012$ ) and radiation dose (as a continuous variable, $\mathrm{P}=0.025$ ). Higher radiation dose and lower NLR were independently associated with better OS (Table 2). The median OS was 8.87 (95\% CI, 7.49-15.31), 15.60 (95\% CI, 10.51-23.19), and 21.85 months (95\% CI, 17.61-30.23) for the patient receiving 50-54.9 Gy, 55-59.9 Gy, $\geq 60 \mathrm{~Gy}$, respectively ( $\mathrm{P}=0.0061)$ (Figure 4).

\section{Failure pattern}

Seventy-nine patients experienced treatment failures. The
Table 2 Multivariate Cox analysis of the risk of death

\begin{tabular}{|c|c|c|}
\hline Variable & Hazard ratio (95\% Cl) & $P$ value \\
\hline \multicolumn{3}{|l|}{ T category } \\
\hline $\mathrm{T} 1-2$ & 1 (reference) & - \\
\hline T3-4 & $0.75(0.29-1.9)$ & 0.547 \\
\hline \multicolumn{3}{|l|}{$\mathrm{N}$ category } \\
\hline $\mathrm{N} 1$ & 1 (reference) & - \\
\hline N2-3 & $1.19(0.72-2.0)$ & 0.488 \\
\hline \multicolumn{3}{|l|}{ Location } \\
\hline Cervical & 1 (reference) & - \\
\hline Upper thoracic & $0.74(0.33-1.6)$ & 0.461 \\
\hline Middle thoracic & $0.70(0.32-1.5)$ & 0.363 \\
\hline Lower thoracic & $0.71(0.32-1.6)$ & 0.403 \\
\hline \multicolumn{3}{|l|}{ Performance status } \\
\hline ECOG 0-1 & 1 (reference) & - \\
\hline ECOG 2-4 & $1.10(0.58-2.1)$ & 0.767 \\
\hline Age & $1.00(0.98-1.0)$ & 0.949 \\
\hline \multicolumn{3}{|l|}{ Sex } \\
\hline Male & 1 (reference) & - \\
\hline Female & $1.78(0.89-3.6)$ & 0.104 \\
\hline GTV & $1(1.00-1.0)$ & 0.908 \\
\hline RT dose & $1.00(1.00-1.0)$ & $0.025^{\star}$ \\
\hline NLR & $1.05(1.01-1.1)$ & $0.012^{*}$ \\
\hline \multicolumn{3}{|l|}{ SCLN group } \\
\hline No SCLN mets & 1 (reference) & 0.837 \\
\hline SCLN mets & $0.95(0.61-1.5)$ & \\
\hline \multicolumn{3}{|c|}{$\begin{array}{l}\text { *, P<0.05. Cl, confidence interval; ECOG, Eastern Cooperative } \\
\text { Oncology Group; RT, Radiotherapy; NLR, Neutrophil-to- } \\
\text { lymphocyte ratio; SCLN mets, supraclavicular lymph node } \\
\text { metastasis; GTV, gross tumor volume. }\end{array}$} \\
\hline
\end{tabular}

median time to failure was similar between patients with or without SCLN metastasis (Figure 5). Among all patients, 27 (18.9\%) developed locoregional failure first, while 41 developed distant failure first $(28.7 \%)$. There were 11 patients who experienced synchronous locoregional and distant failure as the first failure event $(7.7 \%)$. There was no significant difference in failure patterns between patients with SCLN metastasis and those without SCLN metastasis $(\mathrm{P}=0.7999)$. The failure patterns are shown in Table 3. 


\section{Discussion}

The current study demonstrated that SCLN metastasis was not associated with poorer survival in patients with unresectable ESCC treated with definitive chemoradiation. The hazard ratio for OS was $0.95(0.61-1.5)$ compared to patients with no SCLN metastases (Table 2).

Lymph node metastasis is one of the most important

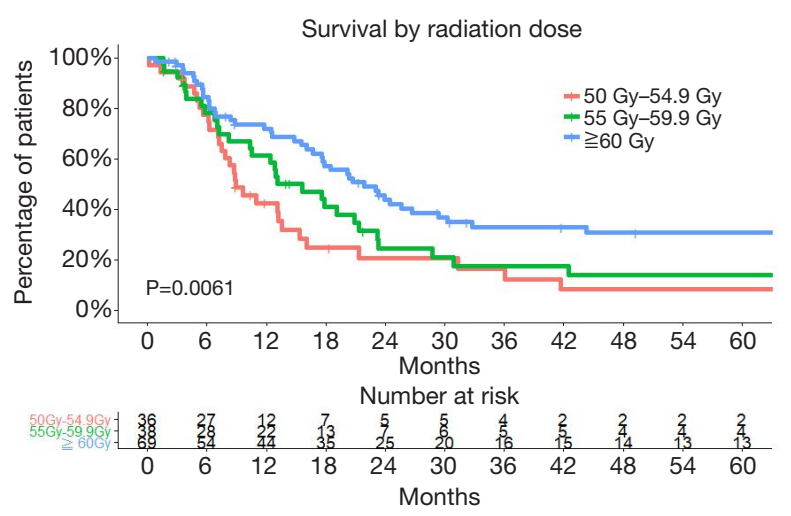

Figure 4 Overall survival according to the radiation therapy dose.

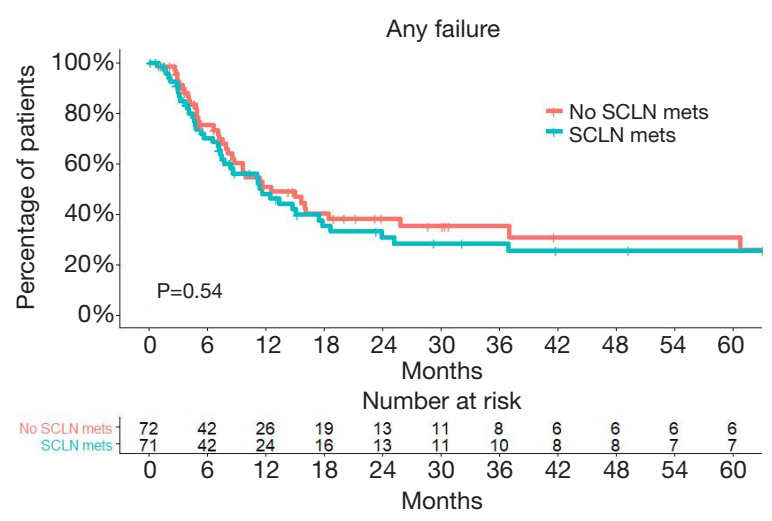

Figure 5 Time to any failure of node-positive esophageal cancer patients with or without supraclavicular lymph node metastasis. SCLN metastasis, supraclavicular lymph node metastasis. prognostic factors in ESCC. Extensive, longitudinal, and intramural interconnecting systems of the lymphatic system can be found within the esophagus. The entire esophagus may have a potential risk of lymphatic involvement (11). The reported incidence of nodal spread is $10 \%$ to $21 \%$ for T1 lesions and increases to $33 \%$ to $69 \%$ for T2 lesions (12). Unlike nasopharyngeal carcinoma or Hodgkin lymphoma, which appear to spread or grow by contiguous extension, skipped metastatic lymph nodes without regional lymph node involvement is not uncommon.

A surgical series from Japan showed that $14.5 \%$ of 1,309 thoracic esophageal cancer patients had pathologic SCLN metastases when they underwent 3-field dissection (7). Another study from China showed the incidence of SCLN metastases was $11.1 \%$ for patients with upper thoracic tumors (13). For patients with esophageal carcinoma, there is great controversy regarding the impact of SCLN metastasis. The two most widely used staging systems, including the AJCC and the Japanese guidelines, have different views on whether SCLN is a regional lymph node. Moreover, the classification of SCLN metastasis into regional or distant metastasis changed over time in different versions of the AJCC staging system. The outcomes of patients who underwent chemoradiation are poorly presented in the AJCC staging system, which is largely based on the surgical series. Clinical staging determines the treatment goal and further influences the treatment strategies.

Several studies have also evaluated the role of SCLN metastasis in patients receiving definitive chemoradiation. Jeene et al. investigated the relationship between SCLN metastasis and the impact on OS. There were $49.7 \%$ with squamous cell carcinoma and $46.7 \%$ with adenocarcinoma in their cohort. The median OS was 23.6 and 17.1 months in patients with and without SCLN metastasis, respectively. SCLN metastasis was not a significant prognostic factor in the multivariate analysis (13). Another study from Taiwan included 369 patients with locally advanced ESCC, 70 of which had SCLN metastasis. Although patients with

Table 3 Failure pattern

\begin{tabular}{lllll}
\hline Variable & Local failure & Distant failure & Synchronous failure & No failure \\
\hline SCLN metastasis & $15(21.13 \%)$ & $22(30.99 \%)$ & $4(5.63 \%)$ & $30(42.25 \%)$ \\
No SCLN metastasis & $12(16.67 \%)$ & $19(26.39 \%)$ & $7(9.72 \%)$ & $34(47.22 \%)$ \\
Total & 27 & 41 & 11 & 64 \\
\hline
\end{tabular}

Data in parentheses are percentages. 
SCLN metastasis had a higher rate of N3 status, it had no significant impact on OS (14). Xu et al. used propensity score matching analysis in 751 patients. They found that SCLN metastasis in middle or lower thoracic esophageal cancer resulted in worse outcomes in distant-metastatic free survival and progression-free survival (15).

The outcome of patients in our cohort was comparable to that reported in the literature. One study showed that the median OS was 14.1 months and the 5-year survival was $27 \%$ in the chemoradiation group (16). Other reports of ESCC patients treated with definitive chemoradiation in the modern era showed a median OS of approximately 16.6-17.5 months. However, in contrast to our study that included only node-positive patients, node-negative patients constituted a significant proportion of the patient population in these studies (13-15).

One meta-analysis of 13 studies revealed an obvious improvement in surgical outcomes after 3 -field lymphadenectomy compared with those after 2 -field lymphadenectomy (17). Three-field lymphadenectomy involved more extensive lymph node dissection in the bilateral supraclavicular regions. A higher 5-year survival rate (hazard ratio, 0.64) was observed in patients receiving 3 -field lymphadenectomy, especially for patients with nodepositive disease. For patients receiving radiation therapy as an alternative to surgery, elective or therapeutic nodal irradiation extending to the SCLN area is usually applied. Acute toxicity was generally well tolerated and manageable. The absence of a critical structure such as the heart, stomach, or bowel makes dose escalation for gross SCLN node more feasible compared to abdominal non-regional lymph nodes. Treating patients with SCLN metastasis with curative intent is crucial to improve outcomes.

One SEER analysis (18) demonstrated that the outcomes of patients with de novo visceral metastasis were poor. The 5-year OS was 4\% in esophageal cancer with distant metastasis at diagnosis. The 5-year OS of the SCLN metastasis group in our cohort was 20.4\% (95\% CI, $12.1-34.5 \%)$, which is considerably higher. In addition, the 5 -year OS survival in our cohort was around $20 \%$ in both groups. For these patients not amenable for surgery due to advanced disease, comorbidity, tumor location, and synchronous malignancy, definitive chemoradiation still makes long-term survival possible. Therefore, we suggest that patients with SCLN metastases should be treated as having regional nodes because of comparable outcomes with other node-positive patients.

Tumor progression, either locoregional or distant, is the major cause of death in patients with esophageal cancer. One study from the MD Anderson Cancer Center (MDACC) found that $50 \%$ of 239 patients receiving definitive chemoradiation presented with local failure, $48 \%$ with distant failure, and $31 \%$ with no failure (19). In our study, 79 of 143 patients (55.2\%) experienced treatment failure. Twenty-eight presented first with local failure only $(28 / 79,35.4 \%)$. The majority of our patients presented with distant failure, emphasizing the importance of further intensified systemic treatment.

Two important prognostic factors, namely NLR and radiation dose, were identified in our multivariate analysis. Various strategies, including dose escalation, have been tested to improve outcomes. Inter group $0123 \mathrm{had}$ established the gold standard radiation dose of $50.4 \mathrm{~Gy}$ for unresectable esophageal cancer (20). Using old 2D technique and concurrent chemotherapy, they demonstrated that a higher radiation dose (64.8 Gy) was not associated with better local regional control or survival. Currently, there is still no evidence that dose escalation improves outcomes. An analysis from the National Cancer Data Base (NCDB) in 2016 (21) identified patients with stage I to III esophageal cancer receiving radiation doses $\geq 50$ Gy between 2004 and 2012. They found no benefit to dose escalation, consistent with the results of the Intergroup 0123 study. However, the histologic subtype of ESCC only accounts for approximately half $(44.5 \%)$ of these patients. The proportion of use of intensity-modulated radiation therapy (IMRT) was approximately $20 \%$. Therefore, the results may not apply to the ESCC subtype and patients treated with the IMRT technique. Advances in treatment planning and machines improve dose conformality and reduce the radiation exposure to normal tissues. One propensity score-based comparison including more than 600 patients revealed that OS, locoregional control, and non-cancer-related death were significantly better with IMRT than with 3D-CRT. Retrospective data from Zhang et al. (22) suggested a positive correlation between radiation dose and locoregional control. Other published results with modern radiotherapy techniques have shown benefits from radiotherapy dose escalation (23). Our study showed that dose escalation was associated with better OS. The finding was still significant when we excluded patients with cervical esophageal cancer. A phase 1/2 trial (24) of dose-escalated simultaneous integrated boost radiotherapy with concurrent chemotherapy for locally advanced esophageal cancer demonstrated good tolerability and local control with 63 Gy in 28 fractions. Dose escalation may be considered 
in the modern era with the hope of improving outcomes for patients with esophageal cancer.

Cancer-associated inflammation has been shown to be a key determinant of disease progression and survival in most cancers, including esophageal cancer. NLR is widely reported as a timesaving, economical, repeatable and routine inflammation-based prognostic indicators. However, there is no widely accepted optimal cutoff value yet for this factor (other studies range from 2-5) (25). Furthermore, several disease conditions may affect NLR, including essential hypertension, acute coronary syndromes, valvular heart diseases, renal diseases, liver diseases, inflammatory diseases, and infections. Some medications, such as antibiotics, antidiabetic and antihypertensive drugs, and cancer chemotherapy may also affect $\operatorname{NLR}(26,27)$. In our multivariate study, higher NLR was also associated with poor outcome. The interaction of the immune system with disease prognosis and treatment choice needs further exploration.

The multivariate analysis in our study demonstrated that although $\mathrm{T}$ and $\mathrm{N}$ status are important prognostic factors in ESCC, they are not associated with the OS. Although an underpowered significance could not be excluded in the analysis, several confounding factors and comorbidities of this population may lead to a negative result. A similar finding was observed in another study from MDACC including 124 ESCC patients receiving definitive chemoradiation (28). The depth of tumor invasion in the esophageal wall and lymph node involvement were correlated with survival in surgical series (29-31). The results from the non-surgical studies might reflect the limited accuracy of the usual staging evaluation including spiral CT scan of the chest and abdomen, esophagogastroduodenoscopy, endoscopic ultrasound (EUS), and PET-CT scan.

The limitations of our study include its retrospective nature and single institution experience. Not all patients underwent EUS or mediastinoscopy for pathologic confirmation of lymph node involvement before treatment. To reduce selection bias, patients who did not fit the inclusion criteria were excluded, resulting in a relatively small sample size. The strength of our study is that we included detailed pretreatment information in multivariate analysis. All the patients in our study received PETCT as part of the initial staging workup. One study has revealed a $14 \%$ incremental effect of PET-CT scan on the diagnostic accuracy in the initial staging of esophageal cancer compared with CT scan alone. They found PET scan with FDG had 32\% sensitivity, 99\% specificity, and $93 \%$ accuracy for individual lymph node group evaluation $(32,33)$. The high specificity and positive predictive value of PET-CT helps in lymph node staging evaluation. Current National Comprehensive Cancer Network (NCCN) guidelines suggest PET-CT evaluation for all patients if there is no evidence of M1 disease in esophageal cancer. The importance of tissue diagnosis in diagnosing SCLN metastasis is undoubtfully important. However, invasive procedures are associated with potential morbidity and relatively high cost, and ideally would be reserved only for those patients for whom questions remain after comprehensive noninvasive imaging. The outcome of patients with SCLN metastasis was similar to that of biopsy and without biopsy in our cohort. We believe that our report could serve as a hypothesis generating study and make us to reconsider the role of SCLN metastasis in patients with esophageal cancer.

\section{Conclusions}

ESCC with SCLN metastasis is not independently associated with poorer survival in patients treated with chemoradiation. Curative intent treatment should be given to these patients. In addition, NLR and higher radiation dose might have a significant impact on the outcome.

\section{Acknowledgments}

We are thankful for the efforts of every member of the esophageal multidisciplinary team.

Funding: None.

\section{Footnote}

Reporting Checklist: The authors have completed the STROBE reporting checklist. Available at http://dx.doi. org/10.21037/tro-20-45

Conflicts of Interest: All authors have completed the ICMJE uniform disclosure form (available at http://dx.doi. org/10.21037/tro-20-45). The authors have no conflicts of interest to declare.

Ethical Statement: The authors are accountable for all aspects of the work in ensuring that questions related to the accuracy or integrity of any part of the work are appropriately investigated and resolved. The study was 
conducted in accordance with the Declaration of Helsinki (as revised in 2013). The study was approved by the institutional ethics board (No.: 20190220A). All the patients have given a consent for medical record collection before receiving treatment. There is no specified informed consent for this study. All the information obtained only includes routine clinical practice. No additional management was done for patients who were enrolled in this study.

Open Access Statement: This is an Open Access article distributed in accordance with the Creative Commons Attribution-NonCommercial-NoDerivs 4.0 International License (CC BY-NC-ND 4.0), which permits the noncommercial replication and distribution of the article with the strict proviso that no changes or edits are made and the original work is properly cited (including links to both the formal publication through the relevant DOI and the license). See: https://creativecommons.org/licenses/by-nc-nd/4.0/.

\section{References}

1. Bray F, Ferlay J, Soerjomataram I, et al. Global cancer statistics 2018: GLOBOCAN estimates of incidence and mortality worldwide for 36 cancers in 185 countries. CA Cancer J Clin 2018;68:394-424.

2. Castoro C, Scarpa M, Cagol M, et al. Nodal metastasis from locally advanced esophageal cancer: how neoadjuvant therapy modifies their frequency and distribution. Ann Surg Oncol 2011;18:3743-54.

3. Dresner SM, Lamb PJ, Bennett MK, et al. The pattern of metastatic lymph node dissemination from adenocarcinoma of the esophagogastric junction. Surgery 2001;129:103-9.

4. Li H, Zhang Y, Cai H, et al. Pattern of lymph node metastases in patients with squamous cell carcinoma of the thoracic esophagus who underwent three-field lymphadenectomy. Eur Surg Res 2007;39:1-6.

5. O'Sullivan B LW, Haughey BH, et al. AJCC Cancer Staging Manual. 8th ed. Springer 2017:113-22.

6. Japan Esophageal S. Japanese Classification of Esophageal Cancer, 11th Edition: part I. Esophagus 2017;14:1-36.

7. Tachimori Y, Ozawa S, Numasaki H, et al. Supraclavicular node metastasis from thoracic esophageal carcinoma: A surgical series from a Japanese multi-institutional nationwide registry of esophageal cancer. J Thorac Cardiovasc Surg 2014;148:1224-9.

8. Lee PC, Port JL, Paul S, et al. Predictors of longterm survival after resection of esophageal carcinoma with nonregional nodal metastases. Ann Thorac Surg 2009;88:186-92; discussion 192-3.

9. Lerut T, Nafteux P, Moons J, et al. Three-field lymphadenectomy for carcinoma of the esophagus and gastroesophageal junction in $174 \mathrm{R} 0$ resections: impact on staging, disease-free survival, and outcome: a plea for adaptation of TNM classification in upper-half esophageal carcinoma. Ann Surg 2004;240:962-72; discussion 972-4.

10. Burke TW, Heller PB, Hoskins WJ, et al. Evaluation of the scalene lymph nodes in primary and recurrent cervical carcinoma. Gynecol Oncol 1987;28:312-7.

11. Rosenberg JC, Franklin R, Steiger Z. Squamous cell carcinoma of the thoracic esophagus: an interdisciplinary approach. Curr Probl Cancer 1981;5:1-52.

12. Siewert JR, Stein HJ, Feith M, et al. Histologic tumor type is an independent prognostic parameter in esophageal cancer: lessons from more than 1,000 consecutive resections at a single center in the Western world. Ann Surg 2001;234:360-7.

13. Jeene PM, Versteijne E, van Berge Henegouwen MI, et al. Supraclavicular node disease is not an independent prognostic factor for survival of esophageal cancer patients treated with definitive chemoradiation. Acta Oncol 2017;56:33-8.

14. Chen YH, Lu HI, Lo CM, et al. The clinical impact of supraclavicular lymph node metastasis in patients with locally advanced esophageal squamous cell carcinoma receiving curative concurrent chemoradiotherapy. PLoS One 2018;13:e0198800.

15. Xu HY, Wu SX, Luo HS, et al. Analysis of definitive chemo-radiotherapy for esophageal cancer with supraclavicular node metastasis based on CT in a single institutional retrospective study: a propensity score matching analysis. Radiat Oncol 2018;13:200.

16. Herskovic A, Martz K, al-Sarraf M, et al. Combined Chemotherapy and Radiotherapy Compared With Radiotherapy Alone in Patients With Cancer of the Esophagus. N Engl J Med 1992;326:1593-8.

17. Ye T, Sun Y, Zhang Y, et al. Three-field or two-field resection for thoracic esophageal cancer: a meta-analysis. Ann Thorac Surg 2013;96:1933-41.

18. Kim E, Koroukian S, Thomas CR Jr. Conditional Survival of Esophageal Cancer: An Analysis from the SEER Registry (1988-2011). J Thorac Oncol 2015;10:1490-7.

19. Welsh J, Settle SH, Amini A, et al. Failure patterns in patients with esophageal cancer treated with definitive chemoradiation. Cancer 2012;118:2632-40.

20. Minsky BD, Pajak TF, Ginsberg RJ, et al. INT 0123 
(Radiation Therapy Oncology Group 94-05) phase III trial of combined-modality therapy for esophageal cancer: high-dose versus standard-dose radiation therapy. J Clin Oncol 2002;20:1167-74.

21. Brower JV, Chen S, Bassetti MF, et al. Radiation Dose Escalation in Esophageal Cancer Revisited: A Contemporary Analysis of the National Cancer Data Base, 2004 to 2012. Int J Radiat Oncol Biol Phys 2016;96:985-93.

22. Zhang Z, Liao Z, Jin J, et al. Dose-response relationship in locoregional control for patients with stage II-III esophageal cancer treated with concurrent chemotherapy and radiotherapy. Int J Radiat Oncol Biol Phys 2005;61:656-64.

23. Chen CY, Li CC, Chien CR. Does higher radiation dose lead to better outcome for non-operated localized esophageal squamous cell carcinoma patients who received concurrent chemoradiotherapy? A population based propensity-score matched analysis. Radiother Oncol 2016;120:136-9.

24. Chen D, Menon H, Verma V, et al. Results of a Phase 1/2 Trial of Chemoradiotherapy With Simultaneous Integrated Boost of Radiotherapy Dose in Unresectable Locally Advanced Esophageal Cancer. JAMA Oncol 2019;5:1597-604.

25. Yodying H, Matsuda A, Miyashita M, et al. Prognostic Significance of Neutrophil-to-Lymphocyte Ratio and Platelet-to-Lymphocyte Ratio in Oncologic Outcomes of Esophageal Cancer: A Systematic Review and Metaanalysis. Ann Surg Oncol 2016;23:646-54.

26. Alkhouri N, Morris-Stiff G, Campbell C, et al. Neutrophil

doi: $10.21037 /$ tro-20-45

Cite this article as: Yen JH, Jen CW, Huang TT, Tsai YC, Cheng SHC. Association of supraclavicular node metastasis with survival in node positive esophageal squamous cell carcinoma patients treated using definitive chemoradiation. Ther Radiol Oncol 2020;4:28. to lymphocyte ratio: a new marker for predicting steatohepatitis and fibrosis in patients with nonalcoholic fatty liver disease. Liver Int 2012;32:297-302.

27. Balta S, Cakar M, Demirkol S, et al. Higher neutrophil to lymhocyte ratio in patients with metabolic syndrome. Clin Appl Thromb Hemost 2013;19:579.

28. Xi M, Xu C, Liao Z, et al. The impact of histology on recurrence patterns in esophageal cancer treated with definitive chemoradiotherapy. Radiother Oncol 2017;124:318-24.

29. Fujita H, Sueyoshi S, Tanaka T, et al. Prospective nonrandomized trial comparing esophagectomy-followed-bychemoradiotherapy versus chemoradiotherapy-followedby-esophagectomy for T4 esophageal cancers. J Surg Oncol 2005;90:209-19.

30. Berger AC, Farma J, Scott WJ, et al. Complete response to neoadjuvant chemoradiotherapy in esophageal carcinoma is associated with significantly improved survival. J Clin Oncol 2005;23:4330-7.

31. Lim JTW, Truong PT, Berthelet E, et al. Endoscopic response predicts for survival and organ preservation after primary chemoradiotherapy for esophageal cancer. Int J Radiat Oncol Biol Phys 2003;57:1328-35.

32. Sasaki K, Uchikado Y, Okumura H, et al. Role of (18) F-FDG-PET/CT in Esophageal Squamous Cell Carcinoma After Neoadjuvant Chemoradiotherapy. Anticancer Res 2017;37:859-64.

33. Kato H, Miyazaki T, Nakajima M, et al. The incremental effect of positron emission tomography on diagnostic accuracy in the initial staging of esophageal carcinoma. Cancer 2005;103:148-56. 